\title{
Automated and high accuracy out-of-hospital heart diseases early detection system
}

\begin{abstract}
Background: ECG measurement and analysis being performed in-hospital normally check patients with obvious symptoms. Asymptomatic patients, for example, those with silent ischemia, paroxysmal atrial fibrillation and Brugada syndrome are very difficult to detect using in-hospital ECG system. To make matter worst, conventional ECG analysis techniques only focuses in time domain that limits the sensitivity, specificity and accuracy of the interpretation due to ECG signals non-stationary nature. Most recent researches have proposed linear joint time-frequency analysis to overcome these limitations, however, their linear methods limit their accuracy.

Objective: The main objective is to enable automated and high accuracy out-of-hospital ECG analysis for early detection of heart disease.

Materials \& Methods: MIT-BIH ECG databases are used to test the performance of the proposed system. The data is pre-processed for amplitude normalization and frequency resampling. The pre-processed output is then fed to the non-linear joint time-frequency algorithm for analysis. The outcome of the analysis is further classified by the machine learning algorithm. Before the classification can be performed, the intelligent classifier is trained using control data that contains 52 normal and 148 patients data. After that, it is used for classifying 40 normal and 88 patients with ischaemia. The entire processes is automated inside a computer. The main disadvantage of the proposed non-linear joint time-frequency technique together with the intelligent classification is that it requires huge amount of computing system resources (big data problem). To solve this issue the system can be connected to the cloud to further enhance the performance and the accuracy of the system.
\end{abstract}

Results: The analysis is performed on 421632 normal and 863282 patients ECG beats. The results obtained show that the system gives high performance of accuracy, sensitivity and specificity with values of $99.03 \%, 99.59 \%$ and $99.57 \%$, respectively.

Conclusions: The proposed method operates in joint time-frequency in non-linear fashion. Hence it produces more accurate interpretation compared to the conventional time domain and the more recent linear time-frequency techniques. The high accuracy together with the automated capability of the system can help detect heart problems at the early stages even before the patients pay visit to the hospital. 